\title{
Electric-Shock Safeguard System for Maintenance of the Transmission Line Using Virtual Fence and GPS Positioning
}

\author{
Jiaying $\mathrm{Li}^{1}$, Yi Zhang ${ }^{1}$ and Chengzhang Jin ${ }^{2}$ \\ ${ }^{1}$ School of Electrical Engineering, Shanghai University of Electric Power, Shanghai 200090, China \\ ${ }^{2}$ State Grid Shanghai Electric Power Company, Shanghai 200235, China
}

\begin{abstract}
In order to avoid the electric shock accident in the process of line maintenance, this paper designs a set of electric-shock safeguard system using virtual fence and GPS positioning service. The system set up a virtual fence in the server side at first, then it compares fence information with real-time position of the monitor side to complete the judgment of boundary limit and achieve the alarm function, which can ensure the safety of the maintainer.
\end{abstract} GPS

Keywords-electric-shock safeguard; maintenance; virtual fence;

\section{INTRODUCTION}

Maintenance of the transmission line is an essential way to ensure the safety of power grid. There are quite a few lines using double-loop or multi-loop construction method. When a section of the line needs to repair blacks out, the rest of the line may be alive. If the maintainer enters the electrification area without the alarm information in time, it will cause an electric shock accident.

For this safety problem, the power industry is using a series of methods, such as induced voltage alarm device, line name assignment, color code distinction between lines, care workers in place. Although these methods in a way reduce accident, it doesn't work very well because of the complexity of the working site environment, and fails to combine the technical safeguard with tradition method. Therefore, seeking a newly effective electric-shock safeguard of the line maintenance has become a urgent affair to improve maintenance safety.

The traditional electronic fence, such as pulse electronic fence, has applied in substation security system. It not only has the monitor and alarm function, also builds the physical fence corresponding to transmit information and prevent invasion. Recently, with the positioning technology civilianizing, the virtual fence provided by positioning service based on GPS gradually applies to vehicle monitoring, goods tracking, smart cultivation, etc ${ }^{[1-4]}$. Compared with the traditional electronic fence, virtual fence can apply for any circumstance without building a physical fence.

In the application of electric-shock safeguard system, it needs higher positioning accuracy to reach centimeter level track of the maintenance monitor side. Therefore, this paper designs electric-shock safeguard system using geographic information system (GIS) to create a virtual fence and using GPS real time kinematic (GPS-RTK) technology to receive monitor side location, combined 3d location information of centimeter level with the virtual fence information, achieving real-time positioning and overstepping alarm.

\section{The MAIN Frame OF SyStem}

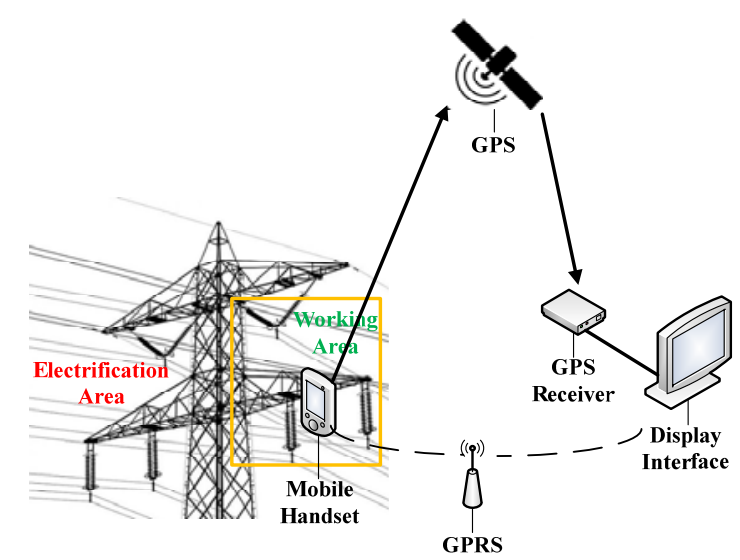

FIGURE I. SYSTEM DRAWING

As Figure I shows, the core technology of the system is the GPS-RTK positioning technology. At first, operator creates a virtual fence, the security working area boundary, on the host by GIS and transmits the fence information into the display interface. Then the processing system completes the judgment of boundary limit by receiving mobile handset real-time location and comparing it to fence information. If the handset equipment crossed the boundary, the system generates alarm command and transmits request to the equipment through GPRS network. 


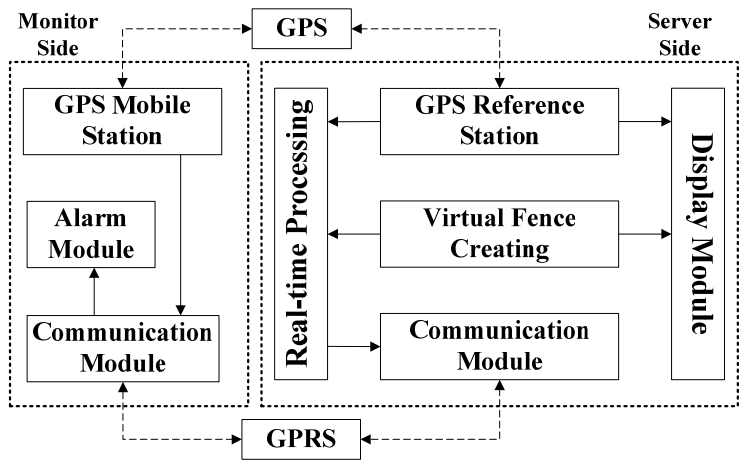

FIGURE II. THE ARCHITECTURE OF SYSTEM

According to the system sketch, considering the actual circumstance of maintenance site, the hardware structure of system is designed as Figure II. The whole system can be divided into the host server side and the mobile monitor side. The monitor side includes GPS mobile station, alarm module and communication module, while the server side includes GPS reference station, virtual fences creating module, real-time processing module, communication module and display module.

The system processes as follows:

Step 1, create the virtual fence according to the maintenance site, and transmit the fence information to real-time processing module and display module at the same time;

Step 2, after maintainer go into the work area carrying mobile monitor equipment, open the GPS positioning;

Step 3, startup the real-time processing module, receive the precise position information of the monitor side using GPS-RTK technology and compare it with the fence information. If maintainer enters areas outside the fence, the server side sends alarm command and monitor side responses it by raising alarm.

Step 4, transmit the position information to the display module.

\section{The Virtual FenCE CREATING Unit}

Because of the difference of maintenance sites, electrification areas should be measured before the positioning system startup. 3d virtual fence is created using GIS on the server side based on the dangerous area.

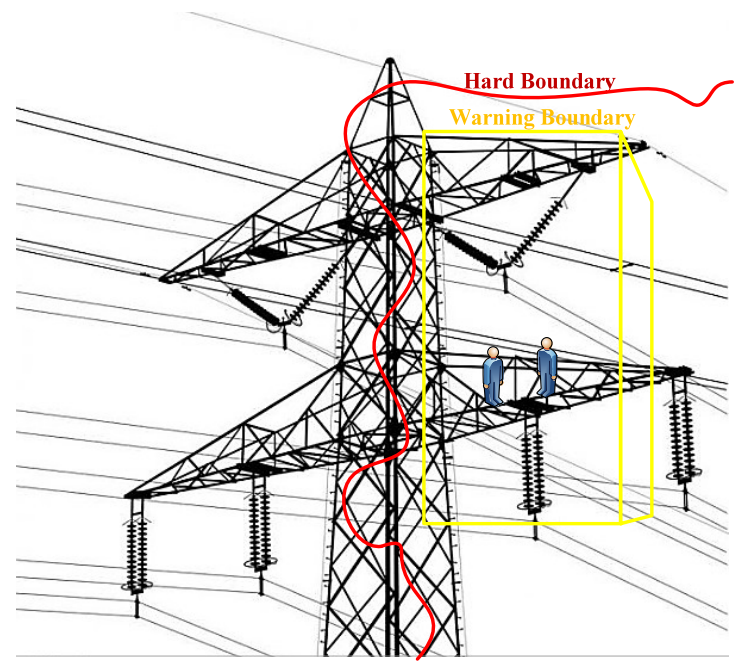

FIGURE III. VIRTUAL FENCE CREATING DRAWING

As is shown in Figure III, the red boundary represents for dangerous area's border, and the yellow boundary represents for warning area's border. Maintainers can work inside the yellow boundary safely. In order to facilitate the judgment of boundary limit, we select warning boundary as a regular cuboid, ensuring the minimum distance between the cuboid and the hard boundary is longer than 1 meter. When the maintainer goes out of the yellow area, mobile equipment alarms. Then when the maintainer crosses over the red border, high frequency alarm information rings and the warning message is sent to the server side.

\section{GPS- RTK POSITIONING UNIT}

GPS-RTK technology is a method using carrier phase real-time dynamic difference technology to measure the precise positioning of object. It builds the GPS receiver and communication platform in a known site called reference station, tracking all of the visible satellite continuously, and transmitting correction data to the mobile station through GPRS. The GPS receiver of mobile station will receive the correction data to get high precision information of mobile station.

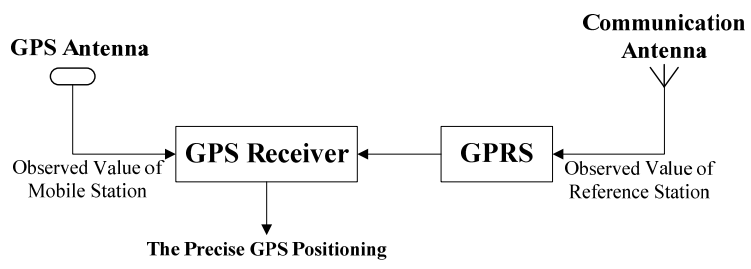

FIGURE IV. GPS MOBILE STATIONG

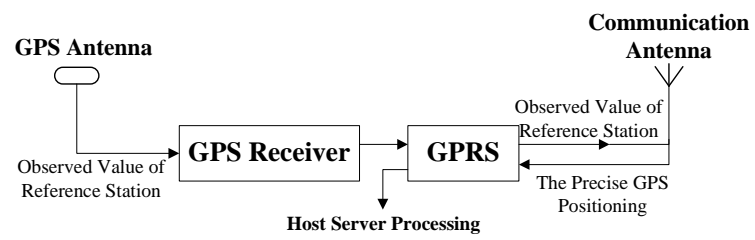

FIGURE V. GPS REFERENCE STATIONG 
As can be seen from the figures, the reference station receives satellite signals using GPS receiver and GPS antenna, and sends correction data to the mobile station through GPRS; the mobile station receives satellite signals and the correction data to get the precise positioning information of centimeter level by computing. Then the positioning information is sent back to the host server through GPRS network, and converted into 3d coordinates prepared for processing and display.

The operating mode of GPRS is designed as point to point or point to multi-point way according to the characteristic of RTK. It just transmits data inside the GPRS network, does not use public IP and port. Therefore, it can ensure the safety and accuracy of the information transmission.

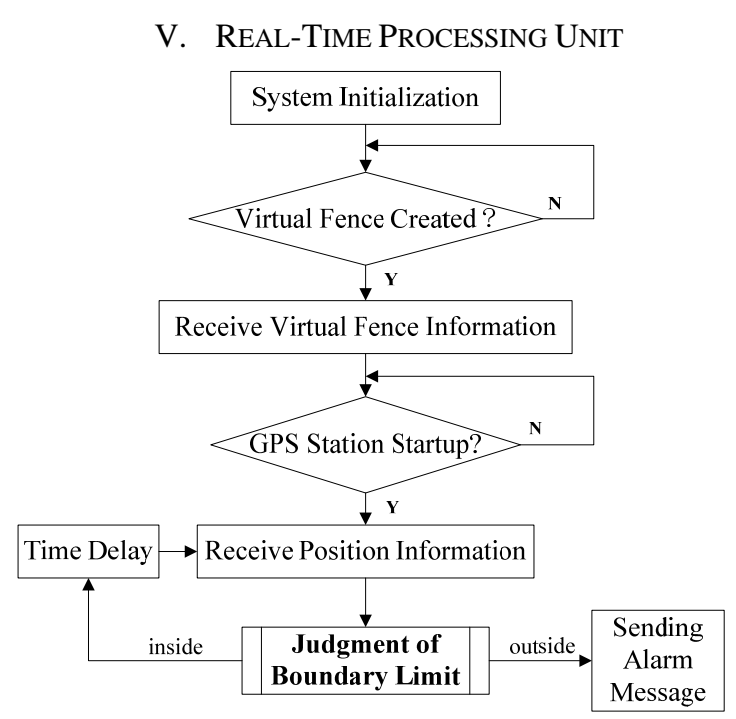

FIGURE VI. FLOW CHART OF PROCESSING

Real-time processing unit mainly includes system initialization, the receiving and displaying of fence information and location information, the judgment of boundary limit, the generation of alarm command. The flow chart of system processing is shown in Figure VI. After the system initialization, program goes into the loop waiting for receiving information. When the system receives the fence information and the first positioning information, it starts the inner cross-border judgment to judge whether the monitor side crosses the yellow area. If the result is fault, it receives positioning information again after 0.5 second delay. Otherwise, it sends alarm command to monitor side, and starts the outer cross-border judgment, namely the judgment of red boundary limit. When the monitor equipment crosses the dangerous border, it sends monitor side a message to ring high frequency alarm, and feedback warning notice to the server side at the same time.

\section{A. The Inner Cross-Border Judgment}

Assuming that mobile monitor equipment is carried on maintainer's waist, maintainer's activity area can limit in a cylinder regarding the equipment point as the center like Figure VII (a), whose radius is 1 meter. On account of the inner yellow boundary is a cuboid and away from the danger zone, the model can be simplified from a 3d motion cylinder to a plane moving point, as shown in Figure VII (b).

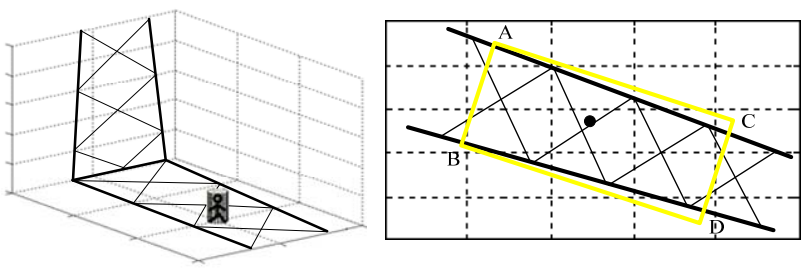

FIGURE VII. THE INNER CROSS-BORDER JUDGENENT DRAWING: (a) 3D MOTION CYLINDER; (b) PLANE MVING POING

We assume the moving point coordinate is $(X, Y, Z)$, and read yellow plane boundary coordinates as $\left(X_{i}, Y_{i}\right), i \in\{A, B, C, D\}$ from fence information when the height is $\mathrm{Z}$. The inner cross-border judgment can be regarded as whether the moving point is outside the rectangle formed by the vertices. Furthermore, considering the actual circumstance of maintenance site that people can only move on the tower's cross arm, whether the point is inside the rectangle is determined to whether the point between the parallel lines of $A B$ and $C D$.

The line equations of $\mathrm{AB}$ and $\mathrm{CD}$ can be obtained

$$
\begin{aligned}
& l_{1}: a_{1} x+b_{1} y+c_{1}=0,\left(X_{A}, Y_{A}\right),\left(X_{B}, Y_{B}\right) \in l_{1} \\
& l_{2}: a_{2} x+b_{2} y+c_{2}=0,\left(X_{C}, Y_{C}\right),\left(X_{D}, Y_{D}\right) \in l_{2}
\end{aligned}
$$

Therefore, it can be judged by $\Delta$ value:

$$
\Delta=\left(a_{1} X+b_{1} Y+c_{1}\right)\left(a_{2} X+b_{2} Y+c_{2}\right)
$$

When $\Delta<0$, the monitor side is inside the yellow boundary. Otherwise, the inner crossing happened.

\section{B. The Outer Cross-Border Judgment}

According to the actual situation, the red boundary can be considered as a non-closed smooth surface. Due to the surface spreads in the space, and its change of two meters height can be neglected, we still regard the monitor side as a moving point.

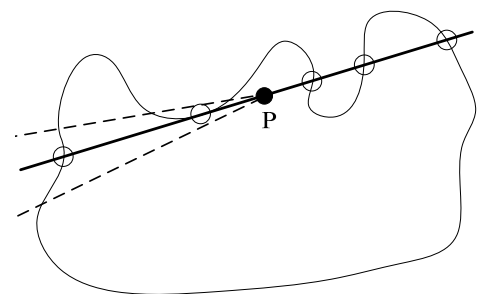

FIGURE VIII. RAY CASTING ALGORITHM

The ray casting algorithm is a computer method to judge a point is inside a closed graph. It counts the number of times a ray starting from a point $\mathrm{P}$ crosses boundary separating it is inside and outside. If this number is odd, then the point is inside. Considering the special circumstances that the ray is just a tangent of the boundary, if the number is even, computer 
creates rays again along with the given direction, which has an angle of $\theta$ degrees from the original direction, to judgment.

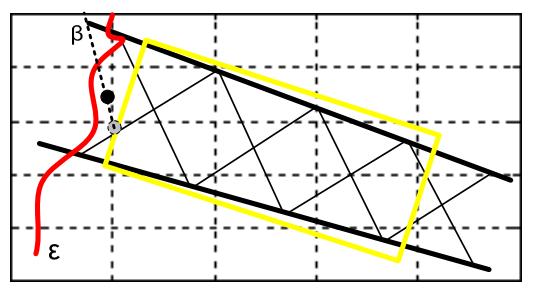

FIGURE IX. THE OUTER CROSS-BORDER JUDGENENT DRAWING

For the non-closed surface, it can use the ray casting principle for the same side judgment, as shown in Figure IX. When inner crossing happened, computer creates a ray named $\beta$ that begins the location at $t$ moment and across the point at $t+1$ moment. If the number of intersections is odd, it regards that the monitor side is inside. Otherwise, if the number is even, it creates a ray which has an angle of $\theta$ degrees from the original direction to judge again. While the number is still even, the outer crossing seems happened.

\section{CONCLUSION}

This paper designs a set of electric-shock safeguard system using virtual fence and GPS positioning service to ensure the safety of maintainers at first. Then we study the algorithm of the judgement of boundary limit to achieve system function.

\section{REFERENCES}

[1] Kaiyuan ZHANG, Rendong YING, Peilin LIU, etc. Design and Development of Beidou Location Based Service Platform [C].The 4th annual Chinese satellite navigation academic conference.2013:10-15.

[2] Ye Lei, Wang Yuanfei. Marine navigation services based on COMPASS (Beidou) and GPS [C]. 2012 Ubiquitous Positioning, Indoor Navigation, and Location Based Service (UPINLBS). 2012: 1-7.

[3] D. A. Vincenzi, B. A. Terwilliger, D. C. Ison, Unmanned aerial system (UAS) human-machine interfaces [C]: New paradigms in command and control, Procedia Manufacturing, Vol. 3, pp. 920-927, 2015.

[4] D.M. Anderson and C.S. Hale, "Animal control system using global positioning and instrumental animal conditioning," Tech. Rep. US Patent 6,232,880, USDA, May 2001. 